\title{
On the Dimensions of Automorphism Groups of Eight-Dimensional Double Loops
}

\author{
By
}

\author{
Richard Bödi, Tübingen
}

(Received 16 February 1993)

\begin{abstract}
Let $\mathscr{D}$ be an eight-dimensional, locally compact, connected double loop. It is proved that the dimension of the automorphism group Aut $\mathscr{D}$ with respect to the compact-open topology is at most 16 .
\end{abstract}

Throughout this paper, let $\mathscr{D}$ denote a locally compact, connected double loop and let $\Gamma$ be a closed subgroup of the full automorphism group of $\mathscr{D}$, which is taken with the compact-open topology. By [14] and [9, XI.8.5], the (covering) dimension of $\mathscr{D}$ is $1,2,4,8$, or, possibly, $\infty$. Excepting the appendix and Lemma (1.1), the dimension of $\mathscr{D}$ is always assumed to be eight. By [2], the group $\Gamma$ is a locally compact transformation group of $\mathscr{D}$ and so the covering dimension coincides with both inductive dimensions (see [18, Thm. 2.1]). The same is true for the double loop $\mathscr{D}$ (see Lemma (3.1) of the appendix). In the case where $\mathscr{D}$ is an eight-dimensional ternary field, H. SALzManN has shown in [21] that either the connected component of $\Gamma$ is isomorphic to the compact exceptional simple Lie group $G_{2}$, or $\operatorname{dim} \Gamma<14$. This result depends on a compactness criterion for the group $\Gamma$ (see [21, (2.2)]). For double loops such a criterion is not yet known.

For a subset $M \subseteq \mathscr{D}$, the smallest closed sub-double-loop of $\mathscr{D}$ containing $M$ is denoted by $\langle M\rangle$. The double loop $\langle M\rangle$ is said to be generated by $M$. If $\langle M\rangle \neq \mathscr{D}$, then $\operatorname{dim}\langle M\rangle \in\{0,1,2,4\}$ by the above result. Note that no example of a locally compact, connected double loop containing a 0 -dimensional double loop is known. We shall call $\mathscr{E}:=\langle 1\rangle$ the prime double loop of $\mathscr{D}$. For any subgroup $\Phi$ of $\Gamma$, we denote by $\mathscr{F}_{\Phi}$ the set of all those elements of $\mathscr{D}$ that are fixed by every automorphism $\varphi \in \Phi$. Clearly, $\mathscr{F}_{\Phi}$ is a closed sub-double-loop of $\mathscr{D}$. If $\Phi$ leaves a sub-double-loop $\mathscr{H}$ of $\mathscr{D}$ invariant, it induces on 
$\mathscr{H}$ an automorphism group $\left.\Phi\right|_{\mathscr{H}}$, see also (3.2) of the appendix. We shall write $\Gamma_{[\mathscr{H}]}$ for the (closed) subgroup of $\Gamma$ that fixes $\mathscr{H}$ pointwise. The one-point compactification $\mathscr{D} \cup\{\infty\}$ of $\mathscr{D}$ is denoted by $\hat{\mathscr{D}}$; it is homotopy equivalent to $\mathbb{S}_{8}$, see [14] and [9, XI.8.5]. All occurring homology groups are assumed to be singular homology groups with coefficient domain $\mathbb{Z}$, whereas the cohomology groups are used in the sense of Alexander-Spanier-Cech (see e.g. [24, Chap. 6, Sect. 4] or $[15$, Chapt. IX, $\S 6]$ ). Reduced (co-)homology groups are written with a tilde on top. When speaking about dimension in general, we always mean the covering dimension dim. Unless stated otherwise, we may assume by the sum theorem (see $[19, \S 3,2.5]$ ) that the group $\Gamma$ is connected, since we are only interested in the topological dimension of $\Gamma$. The center of $\Gamma$ is denoted by $Z(\Gamma)$. We use the symbol $\pi$ for the circle group.

\section{The Dimensions of $\Gamma$-Orbits}

To obtain upper bounds for $\operatorname{dim} \Gamma$, we first have to establish non-trivial upper bounds for the dimensions of $\Gamma$-orbits. The following lemma generalizes a result of $H$. SALZMANN [22].

1.1. Lemma. Let $\operatorname{dim} \mathscr{D} \geqslant 2$. For any element $c \in \mathscr{D}$ which generates a two-dimensional sub-double-loop of $\mathscr{D}$, the inequality

holds.

$$
\operatorname{dim} c^{\Gamma} \leqslant \operatorname{dim} \mathscr{D}-2
$$

Proof. Let $\mathscr{C}:=\langle c\rangle$ be a two-dimensional sub-double-loop. We may assume that $c \notin \mathscr{F}$, else the inequality stated in the lemma holds trivially. Then $\mathscr{F}:=\mathscr{F}_{\mathrm{r}} \cap \mathscr{C}<\mathscr{C}$ and therefore $\operatorname{dim} \mathscr{F}=1$ by $[9$, XI.9.2]. Moreover, the sub-double-loop $\mathscr{C}$ is generated by any element of the set $\mathscr{C} \backslash \mathscr{F}$. Consider the continuous map

$$
\eta: \mathscr{C} \backslash \mathscr{F} \times \Gamma \rightarrow \mathscr{D}:(x, \gamma) \mapsto x^{\gamma^{-1}} .
$$

Because for any element $x \in \mathscr{D}$ the preimage $\eta^{\leftarrow}(x)$ is a closed subset of $\mathscr{C} \backslash \mathscr{F} \times \Gamma$, the monotony theorem (see $[19, \S 6,6.2]$ ) implies that

$$
\operatorname{dim} \eta^{\leftarrow}(x) \leqslant \operatorname{dim}(\mathscr{C} \backslash \mathscr{F} \times \Gamma) \text {. }
$$

Since $\operatorname{dim}\langle c\rangle=2$ and $\operatorname{dim}\langle M\rangle \in\{2,4,8\}$ if $c \in M$, we can find two elements $x, y \in \mathscr{D}$ such that $\mathscr{D}=\langle c, x, y\rangle$. Thus, the stabilizer $\Gamma_{c, x, y}$ is trivial and we conclude that $\operatorname{dim} \Gamma \leqslant 3 \operatorname{dim} \mathscr{D}<\infty$ by repeated application of the dimension formula [10]. Hence, we have $\operatorname{dim}(\mathscr{C} \backslash \mathscr{F} \times$ 
$\times \Gamma)<\infty$. In particular, there is some element $d \in \mathscr{D}$ with $\operatorname{dim} \eta^{\leftarrow}(d)=$ $=\operatorname{dim} \eta:=\sup _{x \in \mathscr{Q}} \operatorname{dim} \eta^{\leftarrow}(x)$. Select arbitrary compact neighborhoods $U \subseteq \mathscr{C} \backslash \mathscr{F}$ and $\Omega=\Omega^{-1} \subseteq \Gamma$, and let $\eta^{*}$ be the restriction of $\eta$ to $U \times \Omega$. Since $U \times \Omega$ is compact, the map $\eta^{*}: U \times \Omega \rightarrow U^{\Omega}$ is a closed surjection, and thus we obtain from $[19, \S 9,2.6]$ the inequality

$$
\operatorname{dim}(U \times \Omega) \leqslant \operatorname{dim} U^{\Omega}+\operatorname{dim} \eta^{*} .
$$

Using the sum theorem $[19, \S 3,2.5]$ and Lemma (3.1) of the appendix, this yields

$$
\operatorname{dim} \mathscr{C}+\operatorname{dim} \Gamma=\operatorname{dim} U+\operatorname{dim} \Omega=\operatorname{dim}(U \times \Omega) \leqslant \operatorname{dim} \mathscr{D}+\operatorname{dim} \eta^{*} .
$$

In particular, we have

$$
\operatorname{dim} \Gamma-\operatorname{dim} \eta^{*} \leqslant \operatorname{dim} \mathscr{D}-2 .
$$

Thus we have to prove the inequality $\operatorname{dim} \eta^{*} \leqslant \operatorname{dim} \Gamma_{c}, \operatorname{since} \operatorname{dim} \Gamma=$ $=\operatorname{dim} \Gamma_{c}+\operatorname{dim} c^{\Gamma}$ holds by [10]. Moreover, it is sufficient to verify that

$$
\operatorname{dim} \eta^{\leftarrow}(d) \leqslant \operatorname{dim} \Gamma_{c},
$$

because we have $\operatorname{dim} \eta^{*} \leqslant \operatorname{dim} \eta=\operatorname{dim} \eta^{\leftarrow}(d)$. Fix an element $b \in d^{\Gamma} \cap$ $\cap \mathscr{C} \backslash \mathscr{F}$. Then the sub-double-loop $\mathscr{C}$ is invariant under an automorphism $\gamma \in \Gamma$ if and only if the element $b^{\gamma}$ lies in $\mathscr{C}$, since $\mathscr{C}$ is generated by any element $x \in \mathscr{C} \backslash \mathscr{F}$. Because a connected twodimensional double loop has at most two continuous automorphisms (see [9, XI.9.3]), the set $\mathscr{C} \cap b^{\Gamma}$ thus contains at most two elements $b$ and $b^{\prime}$. Select $\beta \in \Gamma$ with $b^{\beta}=b^{\prime}$ and choose an automorphism $\delta \in \Gamma$ with $b^{\delta^{-1}}=d$. Setting

$$
A_{1}:=\left\{(b, \gamma) \in\{b\} \times \Gamma \mid b=b^{\delta^{-1} \gamma}\right\}
$$

and

$$
A_{2}:=\left\{\left(b^{\prime}, \gamma\right) \in\left\{b^{\prime}\right\} \times \Gamma \mid b^{\prime}=b^{\delta^{-1} \gamma}\right\}
$$

we obtain

$$
\eta^{\leftarrow}(d)=A_{1} \cup A_{2}
$$

The sets $A_{1}$ and $A_{2}$ have identical dimensions, because they are homeomorphic via the map $(b, \gamma) \mapsto\left(b^{\beta}, \gamma^{\beta}\right)$. Thus the sum theorem yields that $\operatorname{dim} \eta^{\leftarrow}(d)=\operatorname{dim} A_{1}$. The set $A_{1}$ is homeomorphic to $\left\{\gamma \in \Gamma \mid b=b^{\delta^{-1} \gamma}\right\}=\left\{\alpha \in \delta^{-1} \Gamma \mid b=b^{\alpha}\right\} \approx \Gamma_{b}$. Finally, we have $\Gamma_{b}=\Gamma_{c}$, because $\mathscr{C}=\langle b\rangle=\langle c\rangle$, and the lemma is proved. 
The next lemma studies the double loop $\mathscr{F}_{\Gamma}$ of fixed elements if $\Gamma$ is a finite (non-connected) elementary abelian group or a torus group of rank two.

1.2. Lemma. Let $\Gamma \cong \mathbb{Z}_{p}^{2}$ for some prime number $p$. Then $\mathscr{F}_{\Gamma}$ is one- or two-dimensional. If $p=2$ and $\operatorname{dim} \mathscr{F}_{\Gamma}=2$, then $\operatorname{dim} \mathscr{F}_{\gamma}=4$ for every $\gamma \in \Gamma \backslash\{1\}$. If $\Gamma \cong \mathbb{T}^{2}$, then $\mathscr{F}_{\Gamma}$ is two-dimensional.

Proof. Let $\Gamma \cong \mathbb{Z}_{p}^{2}$. Then the fix-double-loop $\mathscr{F}_{\Gamma}$ is connected by [3, (3.3)]. If $\mathscr{F}_{\Gamma}$ would be four-dimensional, all elements of $\Gamma$ would fix $\mathscr{F}_{\Gamma}$ pointwise which is impossible by $\left[6\right.$, p. 262]. Thus $\mathscr{F}_{\Gamma}$ is either one- or two-dimensional. Now let $p=2$ and $\operatorname{dim} \mathscr{F}_{\Gamma}=2$. Let $a, b$, and $c$ denote the dimensions of the fix-double-loops of the three elements in $\Gamma \backslash\{1\}$. Then $a, b, c \in\{2,4\}$. By [3, (3.2)] we may apply [4, Chapt. XIII, $\S 3$, Th. 2.3] and obtain the relation $a+b+c=12$ which implies that $a=b=c=4$. Finally, let $\Gamma \cong \mathbb{T}^{2}$. Then $\Gamma$ contains an elementary abelian subgroup of rank two and thus $\mathscr{F}_{\Gamma}$ is one- or twodimensional. If $\operatorname{dim} \mathscr{F}_{\Gamma}=1$, using the notation from above, we obtain $a+b+c=10$. Hence, we may assume that $a=2$. Since $\Gamma$ is a connected abelian group, it must fix the corresponding two-dimensional fix-double-loop pointwise, which is a contradiction. Hence we have $\operatorname{dim} \mathscr{F}_{\Gamma}=2$.

1.3. Lemma. If $\operatorname{dim} \mathscr{F}_{\Gamma}=0$, then there exists an element $c \in \mathscr{D} \backslash \mathscr{F}_{\Gamma}$ with $\operatorname{dim} c^{\Gamma} \leqslant 7$.

Proof. Suppose that $\operatorname{dim} c^{\Gamma}=8$ for every element $c \in \mathscr{D} \backslash \mathscr{F}_{\Gamma}$. Then $\Gamma$ acts transitively on the complement $\mathscr{D} \backslash \mathscr{F}_{\Gamma}$ by $[3,(3.4)]$, the double loop $\mathscr{D}$ is a topological manifold homeomorphic to $\mathbb{R}^{8}([20,7.12]$ and $[14,5.2])$, and the group $\Gamma$ is a Lie group by $[16,(6.3)]$. Moreover, the orbit $c^{\Gamma}$ is homeomorphic to the homogeneous space $\Gamma / \Gamma_{c}$ by $[3$, (3.1)]. Since the complement $\mathscr{D} \backslash \mathscr{F}_{\Gamma}$ is simply connected by [1], this implies that $\Gamma / \Gamma_{c}$ is simply connected as well. Applying Alexander duality to $\hat{\mathscr{D}}$ and $\hat{\mathscr{F}}_{\Gamma}$, we obtain for $0 \leqslant k \leqslant 7$ the isomorphisms

$$
\tilde{\mathbf{H}}_{k}\left(\Gamma / \Gamma_{c}\right) \cong \tilde{\mathbf{H}}_{k}\left(c^{\Gamma}\right) \cong \tilde{\mathbf{H}}_{k}\left(\mathscr{D} \backslash \tilde{F}_{\Gamma}\right) \cong \tilde{\mathbf{H}}_{k}\left(\hat{\mathscr{D}} \backslash \hat{\mathscr{F}}_{\Gamma}\right) \cong \tilde{\mathbf{H}}^{7-k}\left(\hat{\mathscr{F}}_{\Gamma}\right)
$$

Note that for formulating Alexander duality for non-manifolds $\hat{\mathscr{F}}_{\Gamma}$ we have to take Alexander-Spanier cohomology groups, see e.g. [15, Th. 6.6, p. 222-223] or [7, VIII, 8.15]. Hence we have $\widetilde{\mathbf{H}}_{k}\left(\Gamma / \Gamma_{c}\right)=0$ if $0 \leqslant k \leqslant 6$ and $\mathbf{H}_{7}\left(\Gamma / \Gamma_{c}\right)=\bigoplus^{\kappa} \mathbb{Z}$, because $\hat{\mathscr{F}}_{\Gamma}$ is either finite or homeomorphic to the Cantor set, see $\left[9\right.$, XI.1.5]. In any case, $\hat{\mathscr{F}}_{\Gamma}$ contains 
at least three path components (namely the singletons 0,1 , and $\infty$ ), which implies that $\kappa \geqslant 2$. Since the quotient $\Gamma / \Gamma_{c}$ is simply connected, we may apply the Hurewicz isomorphy theorem (see $[24, \S 7$, Sect. 5, Th. 4]) and obtain the relation $\pi_{7}\left(\Gamma / \Gamma_{c}\right) \cong \mathbf{H}_{7}\left(\Gamma / \Gamma_{c}\right)=\bigoplus^{k} \mathbb{Z}$. Thus, we have the following part of the long homotopy sequence

$$
\pi_{7}(\Gamma) \stackrel{\alpha}{\longrightarrow} \bigoplus^{\kappa} \mathbb{Z} \stackrel{\beta}{\longrightarrow} \pi_{6}\left(\Gamma_{c}\right) .
$$

According to our general assumption that $\Gamma$ is connected, the maximal compact subgroup $K$ of $\Gamma$ is a connected subgroup of $\operatorname{Spin}_{3} \mathbb{R}$, else $\Gamma$ would contain an elementary abelian subgroup of rank two and hence $\mathscr{F}_{\Gamma}$ would be connected by Lemma 1.2. In particular, the group $\mathrm{K}$ is either trivial or isomorphic to one of the groups $\mathbb{\nabla} \approx \mathbb{S}_{1}$ or $\operatorname{Spin}_{3} \mathbb{R} \approx \mathbb{S}_{3}$. By the Malcev-Iwasawa theorem [12, Th. 13, p. 549], the groups $\Gamma$ and $K$ are homotopy equivalent. This implies that the homotopy group $\pi_{7}(\Gamma)$ is finite, see $[24$, Chapt. 9, Sect. 7, Th. 7]. Moreover, the stabilizer $\Gamma_{c}$ is also connected, because the quotient space $\Gamma / \Gamma_{c}$ is simply connected [17, Chapt. $2, \S 8$, Cor. 1]. As before, this implies that $\Gamma_{c}$ is homotopy equivalent to $\mathbb{S}_{1}, \mathbb{S}_{3}$, or to a one-point space. Hence, the group $\pi_{6}\left(\Gamma_{c}\right)$ is also finite, see again [24, Chapt. 9, Sect. 7, Th. 7]. Now, the exactness of the sequence above implies that the kernel of $\beta$ is infinite, which contradicts the fact that the image of $\alpha$ is finite.

\section{Upper Bounds for $\operatorname{dim} \Gamma$}

2.1. Lemma. Let $\Phi$ and $\Psi$ be non-trivial connected subgroups of $\Gamma$ which centralize each other. Then, both groups are at most eightdimensional or one of them is at most four-dimensional.

Proof. If $\mathscr{F}_{\Phi} \neq \mathscr{F}_{\Psi}$, then $\operatorname{dim} \mathscr{F}_{\Phi}=\operatorname{dim} \mathscr{F}_{\Psi}=4$ by Lemma 3.3, and hence by [10], both groups $\Phi$ and $\Psi$ are at most eight-dimensional. Thus in the sequel we may assume that $\mathscr{F}_{\Phi}=\mathscr{F}_{\Psi}=: \mathscr{F}$.

Case 1. For each $c \in \mathscr{D} \backslash \mathscr{F}$ we have $\left\langle c^{\Phi}\right\rangle \neq \mathscr{D} \neq\left\langle c^{\Psi}\right\rangle$. Then $\operatorname{dim}\left\langle c^{\Phi}\right\rangle=\operatorname{dim}\left\langle c^{\Psi}\right\rangle=4$ by [9, XI.8.5, XI.9.3] and, in particular, we have $\operatorname{dim} c^{\Phi} \leqslant 4$ and $\operatorname{dim} c^{\Psi} \leqslant 4$ for every $c \in \mathscr{D} \backslash \mathscr{F}$. Since the groups $\Phi$ and $\Psi$ commute, the stabilizer $\Phi_{c}$ fixes the sub-double-loop $\left\langle c^{\Psi}\right\rangle$ pointwise and thus acts freely on the complement $\mathscr{D} \backslash\left\langle c^{\Psi}\right\rangle$. Hence we have $\operatorname{dim} \Phi_{c}=\operatorname{dim} d^{\Phi_{c}}+\operatorname{dim} \Phi_{c, d} \leqslant 4+0=4$ for some $d \in \mathscr{D} \backslash\left\langle c^{\Psi}\right\rangle$ 
and consequently $\operatorname{dim} \Phi \leqslant 8$ holds. Interchanging the roles of the groups $\Phi$ and $\Psi$ this also gives $\operatorname{dim} \Psi \leqslant 8$.

Case 2. For all $c \in \mathscr{D} \backslash \mathscr{F}$ we have $\left\langle c^{\Phi}\right\rangle \neq \mathscr{D}$, and there is some $d \in \mathscr{D} \backslash \mathscr{F}$ with $\left\langle d^{\Psi}\right\rangle=\mathscr{D}$. Then the stabilizer $\Phi_{d}$ is trivial, since the groups $\Phi$ and $\Psi$ commute. As in the first case we conclude that $\operatorname{dim} d^{\Phi} \leqslant 4$, and thus $\operatorname{dim} \Phi=\operatorname{dim} d^{\Phi} \leqslant 4$ holds.

Case 3. There exist elements $c, d \in \mathscr{D} \backslash \mathscr{F}$ such that $\left\langle c^{\Phi}\right\rangle=\mathscr{D}=$ $=\left\langle d^{\Psi}\right\rangle$. As in the second case we have $\Phi_{d}=1=\Psi_{c}$, which immediately. implies by [10] that $\operatorname{dim} \Phi=\operatorname{dim} d^{\Phi} \leqslant 8$ and $\operatorname{dim} \Psi=\operatorname{dim} c^{\Psi} \leqslant 8$.

We first study the case where $\Gamma$ has a connected fix-double-loop.

2.2. Proposition. If $\operatorname{dim} \mathscr{F}_{\Gamma} \geqslant 1$, then $\operatorname{dim} \Gamma \leqslant 16$.

Proof. For $\operatorname{dim} \mathscr{F}_{\Gamma} \geqslant 2$ the assertion of the proposition is trivial by [10], since in this case the double loop $\mathscr{D}$ is generated by $\mathscr{F}_{\Gamma}$ and two additional elements of the complement $\mathscr{D} \backslash \mathscr{F}_{\Gamma}$. Thus let $\operatorname{dim} \mathscr{F}_{\Gamma}=1$. Since by $\left[9\right.$, XI.8.5, XI.9.3] we have $\operatorname{dim}\left\langle c^{\Gamma}\right\rangle \geqslant 4$ for all $c \in \mathscr{D} \backslash \mathscr{F}_{\Gamma}$, we shall distinguish two cases. If $\operatorname{dim}\left\langle c^{\Gamma}\right\rangle=4$ for some $c \in \mathscr{D} \backslash \mathscr{F}_{\Gamma}$, then the group $\Gamma$ acts on $\mathscr{H}:=\left\langle c^{\Gamma}\right\rangle$ and we obtain

$$
\operatorname{dim} \Gamma=\left.\operatorname{dim} \Gamma\right|_{\mathscr{H}}+\operatorname{dim} \Gamma_{[\mathscr{H}]} \leqslant 4+8=12
$$

by Lemma (3.2) of the appendix and [3]. Turning to the second case we have $\left\langle c^{\Gamma}\right\rangle=\mathscr{D}$ for all $c \in \mathscr{D} \backslash \mathscr{F}_{\Gamma}$. If there is an element $c \in \mathscr{D} \backslash \mathscr{F}_{\Gamma}$ such that $\operatorname{dim}\langle c\rangle \geqslant 4$, then there exists another element $d \in \mathscr{D}$ with $\mathscr{D}=\langle c, d\rangle$. Thus the stabilizer $\Gamma_{c, d}$ is trivial and this implies that $\operatorname{dim} \Gamma=\operatorname{dim} c^{\Gamma}+\operatorname{dim} d^{\Gamma_{c}} \leqslant 8+8=16$. Hence, we may assume that $\operatorname{dim}\langle c\rangle=2$ for each $c \in \mathscr{D} \backslash \mathscr{F}_{\Gamma}$ and we conclude that $\operatorname{dim} c^{\Gamma} \leqslant 6$ holds for all $c \in \mathscr{D} \backslash \mathscr{F}_{\Gamma}$ by Lemma 1.1. Now, since $\mathscr{F}_{\Gamma}$ is connected and since $\mathscr{D}$ is generated by $c^{\Gamma}$, the double loop $\mathscr{D}$ is generated by $\mathscr{F}_{\Gamma}$ and at most three additional elements of the orbit $c^{\Gamma}$. This leads to the inequality $\operatorname{dim} \Gamma \leqslant 3 \operatorname{dim} c^{\Gamma} \leqslant 18$. Furthermore, we may assume that $\operatorname{dim} c^{\Gamma}=6$, since we have $\operatorname{dim} \Gamma \leqslant 3 \cdot 5=15$ if $\operatorname{dim} c^{\Gamma} \leqslant 5$. So, for the rest of the proof we may assume that $17 \leqslant \operatorname{dim} \Gamma \leqslant 18$.

Case 1. $\Gamma$ is semi-simple. Since there is no quasi-simple group of dimension 17 or 18 , the inequality $17 \leqslant \operatorname{dim} \Gamma \leqslant 18$ implies that $\Gamma$ is not quasi-simple. Let $Z$ denote the center of $\Gamma$. By Lemma (3.3), either $\mathscr{F}_{\mathbf{Z}}=\mathscr{F}_{\zeta}$ for every $\zeta \in Z \backslash\{\mathbb{1}\}$ or there is some element $\zeta_{0} \in \mathbf{Z} \backslash\{1\}$ with $\operatorname{dim} \mathscr{F}_{\zeta 0}=4$. Suppose that $Z$ has at least three elements. If $\mathscr{F}_{Z}=\mathscr{F}_{\zeta}$ 
for all $\zeta \in \mathbb{Z} \backslash\{\mathbb{1}\}$, by $\left[9, \mathrm{XI} .9 .1, \mathrm{XI}\right.$.9.3] this implies that $\operatorname{dim}\left\langle c^{\mathbf{Z}}\right\rangle \geqslant 4$ for all $c \in \mathscr{D} \backslash \mathscr{F}_{Z}$, and hence $\operatorname{dim} \Gamma=\operatorname{dim} c^{\Gamma}+\operatorname{dim} \Gamma_{\mathfrak{c}} \leqslant 6+6=12$ holds. If, on the other hand, we have $\operatorname{dim} \mathscr{F}_{\zeta}=4$ for some $\zeta \in Z \backslash\{1\}$, then the group $\Gamma$ leaves $\mathscr{F}_{\zeta}$ invariant, which implies that $\operatorname{dim} \Gamma=$ $=\operatorname{dim} \Gamma \mid \mathscr{F}_{\zeta}+\operatorname{dim} \Gamma\left[\mathscr{F}_{\zeta}\right] \leqslant 4+6=10$ by $[3]$. So let $|Z| \leqslant 2$. Then the group $\Gamma$ is a Lie group which has a maximal torus subgroup of dimension at most two (toroidal rank at most two), see [9, XI.9.6]. Hence the group $\Gamma$ has exactly two quasi-simple factors of toroidal rank one, because the universal covering of $\mathrm{SL}_{2} \mathbb{R}$ is excluded by $|Z| \leqslant 2$. But semi-simple Lie groups of toroidal rank one are at most eight-dimensional, and so $\operatorname{dim} \Gamma \leqslant 16$ follows.

Case 2. $\Gamma$ is not semi-simple. Then the group $\Gamma$ contains a minimal connected closed abelian normal subgroup $\Xi \neq \mathbb{1}$ which is either compact or isomorphic as a topological group to a vector group $\mathbb{R}^{t}$. Since $\Xi$ is connected, every orbit $c^{\Xi}$ for $c \in \mathscr{D} \backslash \mathscr{F}_{\Xi}$ generates a subdouble-loop of dimension at least four, see [9, XI.8.5, XI.9.3]. If $\operatorname{dim}\left\langle c^{\Xi}\right\rangle=4$, the stabilizer $\Gamma_{c}$ acts on $\left\langle c^{\Xi}\right\rangle$, and we obtain $\operatorname{dim} \Gamma_{c}=$ $=\left.\operatorname{dim} \Gamma_{c}\right|_{\left\langle c^{\Xi}\right\rangle}+\operatorname{dim} \Gamma_{[\langle c \Xi\rangle]} \leqslant 4+6=10$ by [3] and Lemma (3.2). This implies that $\operatorname{dim} \Gamma \leqslant 10+6=16$.

So for the rest of the proof we may assume that $\left\langle c^{\Xi}\right\rangle=\mathscr{D}$. If $\Xi$ is compact and hence central in $\Gamma$ (see $[11,(26.20)])$, the stabilizer $\Gamma_{c}$ is trivial and thus $\operatorname{dim} \Gamma \leqslant 6$ follows. Hence we may assume that $\Xi$ is a vector group and that the stabilizer $\Gamma_{\mathfrak{c}}$ acts effectively on $\Xi$. Let $\Gamma_{c}^{1}$ denote the connected component of $\Gamma_{c}$ containing the identity and let $\Pi \leqslant \Xi$ denote a minimal $\Gamma_{c}^{1}$-invariant subspace of $\Xi$. Applying Lemma 3.3 to $\Xi$ and $\Pi$, we obtain that either $\mathscr{F}_{\Pi}$ is four-dimensional and $\Gamma_{c}^{1}$ acts on $\mathscr{F}_{\Pi}$, or $c^{\Pi} \neq c$. If $\operatorname{dim} \mathscr{F}_{\Pi}=4$, we conclude as before that $\operatorname{dim} \Gamma_{c} \leqslant 4+6=10$ and so $\operatorname{dim} \Gamma \leqslant 10+6=16$ holds. If $c^{\Pi} \neq c$, then we either have $\operatorname{dim}\left\langle c^{\Pi}\right\rangle=4$, which implies that $\operatorname{dim} \Gamma=\operatorname{dim} c^{\Gamma}+$ $+\operatorname{dim} \Gamma_{c} \leqslant 6+10=16$, or $\left\langle c^{\Pi}\right\rangle=\mathscr{D}$. So it remains to study the case $\left\langle c^{\Pi}\right\rangle=\mathscr{D}$. Then $\Gamma_{c}^{1}$ acts effectively and irreducibly on the vector space $\Pi$, since $\Pi$ is a minimal $\Gamma_{c}^{1}$-invariant subspace of $\Xi$. By $[8,19.14$, 19.17], this implies that $\Gamma_{c}^{1}$ is a linear Lie group whose radical $\Delta$ is a closed connected subgroup of $\mathbb{C}^{\times}$lying in the center of $\Gamma_{c}^{1}$. Let $\Psi$ denote a Levi complement of $\Gamma_{c}^{1}$. By $[9$, XI.9.6] the dimension of the maximal torus of $\Gamma_{c}^{1}$ is at most two. Consequently, if $\Delta \cong \mathbb{C}^{\times}$then the maximal torus of $\Psi$ is at most one. Thus, by the classification of quasi-simple Lie groups we conclude that $\operatorname{dim} \Psi \leqslant 8$. Then $\operatorname{dim} \Gamma_{c}=$ 
$=\operatorname{dim} \Gamma_{c}^{1}=\operatorname{dim} \Psi+\operatorname{dim} \Delta \leqslant 8+2=10$ which yields $\operatorname{dim} \Gamma \leqslant 16$. Hence we may assume that $\operatorname{dim} \Delta \leqslant 1$.

Now, the set $\Pi^{*}:=\left\{\pi \in \Pi \mid c \in \mathscr{F}_{\pi}\right\}$ forms a subgroup of $\Pi$ which is $\Gamma_{c}^{1}$-invariant, since for $\pi \in \Pi, c \in \mathscr{F}_{\pi}$, and $\gamma \in \Gamma$ we have $c^{\gamma} \in \mathscr{F}_{\pi^{\gamma}}$ and thus $c^{\gamma}=c$ implies that $\pi^{\gamma} \in \Pi^{*}$. Hence the set $\Pi^{*}$ is $\Gamma_{c}^{1}$-invariant. Because of $c^{\Pi} \neq c$, we moreover have $\Pi^{*} \neq \Pi$. But the group $\Pi$ is a minimal $\Gamma_{c}^{1}$-invariant subgroup. We conclude that $\Pi^{*}=1$ and hence $c^{\pi} \neq c$ for all $\pi \in \Pi \backslash\{\mathbb{1}\}$. In particular, we have $\operatorname{dim}\left\langle c^{P}\right\rangle \geqslant 4$ for every oneparameter subgroup $P$ of $\Pi$ and $s:=\operatorname{dim} \Pi \leqslant 6$. Now, the stabilizer $\Gamma_{c}^{1}$ either acts transitively on $\Pi$, or there is a one-parameter subgroup $P$ of $\Pi$ such that $\operatorname{dim} \Gamma_{c}^{1} / \Theta<s$, where $\Theta$ is the centralizer of $P$ in $\Gamma_{c}^{1}$. In the first case, $\Gamma_{c}^{1}$ acts transitively on the projective space $\mathscr{P}_{s-1} \mathbb{R}$ of all one-parameter subgroups of $\Pi$. If there is a one-parameter subgroup $\mathrm{P}$ with $\left\langle c^{\mathrm{P}}\right\rangle=\mathscr{D}$, then $\Theta=1$, because $\Theta$ fixes $c$. This leads to

$$
\operatorname{dim} \Gamma_{c}=\operatorname{dim} \Gamma_{c}^{1} / \Theta+\operatorname{dim} \Theta \leqslant s \leqslant 6
$$

and hence we have $\operatorname{dim} \Gamma=\operatorname{dim} \Gamma_{c}+\operatorname{dim} c^{\Gamma} \leqslant 6+6=12$. So we may assume that $\left\langle c^{P}\right\rangle\langle\mathscr{D}$ holds for all one-parameter subgroups $\mathrm{P}$ in $\Pi$. For the rest of the proof let us fix a one-parameter subgroup $P$ in $\Pi$ and let $\Theta$ be its centralizer in $\Gamma_{c}^{1}$. Since $\Theta$ acts freely on the complement $\mathscr{D} \backslash\left\langle c^{P}\right\rangle$, we have $\operatorname{dim} \Theta=\operatorname{dim} d^{\Theta}+\operatorname{dim} \Theta_{d} \leqslant \operatorname{dim} c^{\Gamma}+$ $+0 \leqslant 6$ for every $\left.d \in c^{\Gamma}\right\rangle\left\langle c^{P}\right\rangle$. Such an element $d$ exists, because we have assumed that $\operatorname{dim} c^{\Gamma}=6$. Altogether, we have

$$
\operatorname{dim} \Gamma_{c}=\operatorname{dim} \Gamma_{c}^{1} / \Theta+\operatorname{dim} \Theta \leqslant s+6 .
$$

Thus, in the sequel we may suppose that $s \geqslant 5$. Since $\operatorname{dim} \Delta \leqslant 1$ and $11 \leqslant \operatorname{dim} \Gamma_{c} \leqslant 12$, the Levi-complement $\Psi$ has one of the dimensions 10,11 , or 12 . We will study each of these cases separately by using the classification of quasi-simple Lie groups and their representations.

a) $\operatorname{dim} \Psi=10$. Then $\Psi$ is quasi-simple and locally isomorphic to an orthogonal group $\mathrm{SO}_{5, r} \mathbb{R}$. Since groups locally isomorphic to $\mathrm{SO}_{5, r} \mathbb{R}$ have no irreducible representation of dimension 6 , we conclude that $s \leqslant 5$. The stabilizer $\Gamma_{c}$ has dimension at most 11 , since $\operatorname{dim} \Delta \leqslant 1$. On the other hand, by what we have proved above we have $\operatorname{dim} \Gamma_{c} \geqslant 11$, and hence we know that $\operatorname{dim} \Gamma_{c}=11$. Choose an element $d \in c^{\Gamma} \cap\left\langle c^{\mathrm{P}}\right\rangle \backslash\langle c\rangle$. If $d^{\Gamma_{c}} \subseteq\left\langle c^{\mathrm{P}}\right\rangle$, then $\operatorname{dim} d^{\Gamma_{c}} \leqslant \operatorname{dim}\left\langle c^{\mathrm{P}}\right\rangle=4$ and we conclude that

$$
\operatorname{dim} \Gamma_{c}=\operatorname{dim} d^{\Gamma_{c}}+\operatorname{dim} \Gamma_{c, d} \leqslant 4+\operatorname{dim} \Gamma_{c, d}
$$


For every element $e \in c^{\Gamma} \backslash\left\langle c^{P}\right\rangle$ we have $\mathscr{D}=\langle c, d, e\rangle$ and thus we conclude that $\operatorname{dim} \Gamma_{c, d} \leqslant \operatorname{dim} e^{\Gamma_{c, d}} \leqslant \operatorname{dim} e^{\Gamma}=\operatorname{dim} c^{\Gamma}=6$. Consequently, we obtain $\operatorname{dim} \Gamma_{c} \leqslant 10$, which is a contradiction. Hence, we may assume that the orbit $d^{\Gamma_{c}}$ is not contained in $\left\langle c^{P}\right\rangle$ and therefore we may select an element $e \in d^{\Gamma_{c}} \backslash\left\langle c^{P}\right\rangle$. As before we have $\mathscr{D}=\langle c, d, e\rangle$. Thus, the stabilizer $\Gamma_{c, d, e}$ is trivial, which implies that

$$
11=\operatorname{dim} \Gamma_{c}=\operatorname{dim} d^{\Gamma_{c}}+\operatorname{dim} \Gamma_{c, d}=\operatorname{dim} d^{\Gamma_{c}}+\operatorname{dim} e^{\Gamma_{c, d}} .
$$

Since we have chosen the element $d$ in the orbit $c^{\Gamma}$, we infer that $\operatorname{dim} d^{\Gamma_{c}} \leqslant \operatorname{dim} c^{\Gamma}=6$ and just so from $e \in d^{\Gamma_{c}}$ we get $\operatorname{dim} e^{\Gamma_{c, d}} \leqslant \operatorname{dim} d^{\Gamma_{c}}$. By the equation above, this implies that $\operatorname{dim} e^{\Gamma_{c, d}}=5$ (and $\operatorname{dim} d^{\Gamma_{c}}=6$ ), and we infer that

$$
\operatorname{dim} \Theta=\operatorname{dim} e^{\Theta} \leqslant \operatorname{dim} e^{\Gamma_{c, d}}=5,
$$

because the centralizer $\Theta$ fixes $\left\langle c^{\mathbf{P}}\right\rangle=\langle c, d\rangle$ pointwise. By inequality $(*)$, this yields $\operatorname{dim} \Gamma_{c} \leqslant s+5 \leqslant 10$, which again is a contradiction.

b) $\operatorname{dim} \Psi=11$. In this case, the group $\Psi$ cannot be quasi-simple and, moreover, it is the product of an eight-dimensional quasi-simple group $\Psi_{1}$ and a three-dimensional quasi-simple group $\Psi_{2}$. Since $\Psi$ is a linear group, a maximal torus subgroup of $\Psi$ has dimension at least two. By Lemma 1.2, every involution in $\Psi$ has a four-dimensional double loop of fixed elements. Select an involution $\omega$ in $\Psi$ which is centralized by the factor $\Psi_{1}$. Thus, $\Psi_{1}$ leaves $\mathscr{F}_{\omega}$ invariant. Because $\Psi_{1}$ is quasi-simple, it must either act trivially or with a zero-dimensional kernel on $\mathscr{F}_{\omega}$. By Lemma 3.2 and [3], this implies that $\operatorname{dim} \Psi_{1} \leqslant$ $\leqslant \max \{4,6\}=6$, which is a contradiction.

c) $\operatorname{dim} \Psi=12$. Then $\Delta=1$ and $\Gamma_{c}^{1}=\Psi$ is the product of two sixdimensional quasi-simple groups $\Psi_{1}$ and $\Psi_{2}$, for else $\Psi$ would contain a three-dimensional torus subgroup (note that $\Psi$ is linear), which is impossible by $[9$, XI.9.6]. Moreover, by inequality (*) we have $\operatorname{dim} \Theta=6=s$ and as mentioned above, $\Gamma_{c}^{1}$ acts transitively on $\mathscr{P}_{s-1} \mathbb{R}$. Since $\Psi$ is linear, we can apply the classification of transitive connected linear groups acting on Graßmann manifolds, see [25], e.g. By this classification, $\Gamma_{c}^{1}=\Psi$ has to be a quasi-simple group, which again is a contradiction.

We now turn to the general case where no restrictions on $\mathscr{F}_{\Gamma}$ are presumed. We start with a result about semi-simple groups.

2.3. Proposition. If $\Gamma$ is semi-simple, then $\operatorname{dim} \Gamma \leqslant 16$. 
Proof. The quotient $\Gamma^{*}:=\Gamma / Z(\Gamma)$ is a semi-simple Lie group, $Z(\Gamma)$ is zero-dimensional, and a maximal compact Lie group $\mathrm{K}^{*}$ of $\Gamma^{*}$ is covered by a Lie group $\tilde{K}$ which is contained in the universal covering $\tilde{\Gamma}$ of $\Gamma^{*}$. In general, the group $\tilde{K}$ need not be compact. So let $\tilde{C}$ denote a maximal compact subgroup of $\tilde{\mathrm{K}}$.

Case $1 . \Gamma$ is quasi-simple. Then $\tilde{C}$ is a compact semi-simple Lie group, which is projected onto a compact semi-simple Lie subgroup $C$ of $\Gamma$ with $\operatorname{dim} C=\operatorname{dim} \tilde{C}$. Furthermore, the inequality $\operatorname{dim} C=$ $=\operatorname{dim} \tilde{C} \geqslant \operatorname{dim} \tilde{K}-1=\operatorname{dim} K^{*}-1$ holds. If $\operatorname{dim} \Gamma^{*} \geqslant 14$ then $\operatorname{dim} K^{*} \geqslant$ $\geqslant 6$ by the classification of quasi-simple Lie groups, and thus the group $\Gamma$ contains a compact Lie group of dimension at least five. Consequently, the group $\Gamma$ contains commuting involutions and the assertion follows from Lemma 1.2 and Proposition 2.2.

Case 2. $\Gamma$ is semi-simple, but not quasi-simple. We write $\Gamma$ as a product $\Gamma=\Phi \cdot \Psi \cdot \Delta$, where $\Phi$ and $\Psi$ are non-trivial quasi-simple groups and $\Delta$ is a (possibly trivial) semi-simple group. By (2.1) we have $\operatorname{dim} \Gamma<\infty$, and thus we may assume that $\Phi$ is a quasi-simple factor of $\Gamma$ of maximal dimension. The group $\Delta$ can be written as the product of at most two non-trivial semi-simple factors, because by Lemma 2.1 we have $\operatorname{dim} \Delta \leqslant 8$ (note that $\operatorname{dim} \Phi \cdot \Psi \geqslant 6>4$ ). Furthermore, we may assume that $\operatorname{dim} \Phi \geqslant 6$, since for $\operatorname{dim} \Phi \leqslant 3$ we would have $\operatorname{dim} \Gamma \leqslant$ $\leqslant 4 \cdot 3=12$. Now $\left\langle d^{\Phi}\right\rangle=\mathscr{D}$ must hold for all $d \in \mathscr{D} \backslash \mathscr{F}_{\Phi}$, because the quasi-simple group $\Phi$ acts on $\left\langle d^{\Phi}\right\rangle$ with a zero-dimensional kernel, i.e. the factor $\Phi$ induces a six-dimensional group on $\left\langle d^{\Phi}\right\rangle$, which is impossible by [3] and Lemma 3.2 if $\operatorname{dim}\left\langle d^{\Phi}\right\rangle=4$. So we have $\left\langle d^{\Phi}\right\rangle=$ $=\mathscr{D}$. Set $\Lambda:=\Psi \cdot \Delta$. Since $\Phi$ commutes with $\Lambda$ and because of $\left\langle d^{\Phi}\right\rangle=\mathscr{D}$, the stabilizer $\Lambda_{d}$ must be trivial. Consequently, we have $\operatorname{dim} \Lambda \leqslant 8$. Now assume that $\operatorname{dim} \Gamma \geqslant 17$. This implies that $\operatorname{dim} \Phi \geqslant 9$. Applying Lemma 2.1 to $\Phi$ and $\Lambda$, we conclude that $\operatorname{dim} \Lambda=3$ and hence $\operatorname{dim} \Phi \geqslant 14$. Finally, choose an element $d \in \mathscr{D} \backslash \mathscr{F}_{\Lambda}$. Then $\operatorname{dim}\left\langle d^{\Lambda}\right\rangle \geqslant 4$. If $\left\langle d^{\Lambda}\right\rangle=\mathscr{D}$, then $\operatorname{dim} \Phi \leqslant 8$ (and hence $\operatorname{dim} \Gamma \leqslant 11$ ), since $\Lambda$ and $\Phi$ commute. Finally, if $\operatorname{dim}\left\langle d^{\Lambda}\right\rangle=4$, we obtain a contradiction as before.

2.4. Lemma. A commutative group $\Gamma$ is at most eight-dimensional. If $\Gamma$ is an eight-dimensional commutative group, then it is isomorphic to $\mathbb{R}^{6} \times \mathbb{T}^{2}$. 
Proof. Let $c \in \mathscr{D} \backslash \mathscr{F}$. Then $\operatorname{dim}\left\langle c^{\Gamma}\right\rangle \geqslant 4$. If $\left\langle c^{\Gamma}\right\rangle=\mathscr{D}$, then the stabilizer $\Gamma_{c}$ is trivial, because $\Gamma$ is commutative. Thus we have $\operatorname{dim} \Gamma \leqslant 8$. Suppose that $\operatorname{dim} \Gamma=8$. Then the group $\Gamma$ acts sharply transitively on the complement $\mathscr{D} \backslash \mathscr{F}$. Hence the group $\Gamma$ is a Lie group, and the double loop $\mathscr{D}$ is a topological manifold (compare the proof of Lemma 1.3). Being a connected commutative Lie group, the group $\Gamma$ is isomorphic to a product $\mathbb{R}^{l} \times \mathbb{T}^{8-l}$. Thus the homology groups $\mathbf{H}_{n}(\Gamma)$ of $\Gamma$ vanish for $n \geqslant 2$. Applying Alexander duality to $\mathscr{D}$ and $\hat{\mathscr{F}}_{\Gamma}$ and noting that $\Gamma / \Gamma_{c} \approx c^{\Gamma}=\mathscr{D} \backslash \mathscr{\mathscr { F }}_{\Gamma}=\hat{\mathscr{D}} \backslash \hat{\mathscr{F}}_{\Gamma}$ holds by $[3$, (3.1)], this implies that the cohomology groups $\widetilde{\mathbf{H}}^{n}\left(\hat{\mathscr{F}}_{\Gamma}\right)$ vanish for $0 \leqslant n \leqslant 5$. In particular, the dimension of $\mathscr{F}_{\Gamma}$ is at least six by Lemma 3.1. So we have $\mathscr{F}_{\Gamma}=\mathscr{D}$, because the dimension of a finite-dimensional locally compact connected double loop is either $1,2,4$, or 8 , see $[9$, XI.8.5] and compare also [14]. But then we have $\Gamma=1$, a contradiction. Thus we have $\operatorname{dim} \Gamma \leqslant 7$ if $\left\langle c^{\Gamma}\right\rangle=\mathscr{D}$. Now let $\operatorname{dim}\left\langle c^{\Gamma}\right\rangle=4$ for every $c \in \mathscr{D} \backslash \mathscr{F}_{\Gamma}$. Fix some element $d \in \mathscr{D} \backslash \mathscr{F}_{\Gamma}$ and set $\mathscr{H}:=\left\langle d^{\Gamma}\right\rangle$. Choose an element $e \in \mathscr{D} \backslash \mathscr{H}$. Since $\operatorname{dim} \mathscr{H}=4$, the group $\Gamma_{d}=\Gamma_{[\mathscr{H}]}$ operates freely on $\mathscr{D} \backslash \mathscr{H}$. In particular, the stabilizer $\Gamma_{d}$ acts effectively on the four-dimensional sub-double-loop $\left\langle e^{\Gamma}\right\rangle$. This implies that $\operatorname{dim} \Gamma=$ $=\operatorname{dim} d^{\Gamma}+\operatorname{dim} \Gamma_{d} \leqslant 4+4=8$, since the stabilizer $\Gamma_{d}$ is at most fourdimensional by [3] and Lemma 3.2. Moreover, if $\operatorname{dim} \Gamma=8$, Lemma 2.4 of [3] yields $\Gamma_{d} \cong \mathbb{R}^{3} \times \mathbb{T}$. Interchanging the roles of the elements $d$ and $e$, the same is true for the stabilizer $\Gamma_{e}$. Finally we have $\Gamma=\Gamma_{d} \times \Gamma_{e}$, since $\Gamma_{d} \cap \Gamma_{e}=1$ and $\operatorname{dim} \Gamma_{d}=\operatorname{dim} \Gamma_{e}=4$. This finishes our proof.

2.5. Proposition. If $\operatorname{dim} Z(\Gamma) \geqslant 1$, then $\operatorname{dim} \Gamma \leqslant 16$.

Proof. Let $Z:=Z(\Gamma)$ and choose an element $c \in \mathscr{D} \backslash \mathscr{F}_{Z}$. Since $\operatorname{dim} Z \geqslant 1$, the sub-double-loop $\left\langle c^{z}\right\rangle$ is of dimension at least four (see [9, XI.9.1., XI.9.3]). If $\left\langle c^{\mathrm{Z}}\right\rangle=\mathscr{D}$, the stabilizer $\Gamma_{c}$ is trivial, which implies that $\operatorname{dim} \Gamma=\operatorname{dim} c^{\Gamma} \leqslant \operatorname{dim} \mathscr{D}=8$. So we may assume that $\mathscr{H}:=\left\langle c^{\mathbf{z}}\right\rangle$ is four-dimensional. Then the stabilizer $\Gamma_{c}$ acts freely on the complement $\mathscr{D} \backslash \mathscr{H}$, since $\Gamma_{c}=\Gamma_{[\mathscr{H}]}$. Thus we have $\operatorname{dim} \Gamma_{c} \leqslant 8$ and the assertion $\operatorname{dim} \Gamma=\operatorname{dim} c^{\Gamma}+\operatorname{dim} \Gamma_{c} \leqslant 8+8=16$ follows.

2.6. Theorem. The automorphism group $\Gamma$ of a locally compact connected eight-dimensional double loop $\mathscr{D}$ is at most 16-dimensional.

Proof. By Proposition (2.3) we may assume that the group $\Gamma$ contains a non-trivial connected commutative normal subgroup $\Xi$. If 
$\Xi$ is compact, it is contained in the center of $\Gamma$ and the assertion of the theorem follows by Proposition 2.5. So, for the remainder of the proof we may assume that $\Xi$ is not compact and hence is isomorphic to $\mathbb{R}^{t}$ for some $t>0$. Moreover, by Proposition 2.2, we may assume that $\operatorname{dim} \mathscr{F}_{\Gamma}=0$. Hence, by Lemma 1.3 we may select an element $c \in \mathscr{D} \backslash \mathscr{F}_{\Gamma}$ with $\operatorname{dim} c^{\Gamma} \leqslant 7$. Choose a minimal $\Gamma_{c}^{1}$-invariant subspace $\Pi \leqslant \Xi$. Using the arguments of $[21,(3.3)]$, we may assume that the stabilizer $\Gamma_{c}$ is a Lie group, since otherwise the dimension of $\left\langle c^{\Gamma}\right\rangle$ would be four and then $\operatorname{dim} \Gamma \leqslant 12$ by [3], because the group $\Gamma$ leaves $\left\langle c^{\Gamma}\right\rangle$ invariant. Next, we may assume that $\Xi$ moves the element $c$, since in the other case the fix-double-loop $\mathscr{F}_{\Xi}$ is four-dimensional by Lemma 3.3, and as before we conclude that $\operatorname{dim} \Gamma \leqslant 12$, because $\Gamma$ leaves $\mathscr{F}_{\Xi}$ invariant.

In the following step we shall show that it suffices to consider the case where $\left\langle c^{\Pi}\right\rangle \neq \mathscr{D}$. Assume that $\left\langle c^{\Pi}\right\rangle=\mathscr{D}$ holds. Then $\Gamma_{c}^{1}$ acts effectively and irreducibly on the vector group $\Pi$. Consequently, the group $\Gamma_{c}^{1}$ is a linear Lie group with a radical of dimension at most two, see $[8,19.14,19.17]$. Now the assumption $\operatorname{dim} \Gamma \geqslant 17$ implies that $\operatorname{dim} \Gamma_{c}^{1} \geqslant 10$, and therefore a Levi-complement $\Sigma$ of $\Gamma_{c}^{1}$ is at least eight-dimensional. Being a linear semi-simple Lie group, $\Sigma$ thus contains commuting involutions. Hence, we have $\operatorname{dim} \Gamma \leqslant 16$ by Lemma 1.2 and Proposition 2.2. So we may assume that $\left\langle c^{\Pi}\right\rangle \neq \mathscr{D}$. For the rest of the proof we also suppose that $\operatorname{dim} \Gamma \geqslant 17$. We shall distinguish two cases.

Case 1. The element $c$ is fixed by the vector group $\Pi$. Then the fix-double-loop $\mathscr{F}_{\Pi}$ is four-dimensional by Lemma 3.3, since the element $c$ is moved by the group $\Xi$. Set $\Pi^{*}:=\left(\Xi \cap \Gamma_{c}^{1}\right)^{1}$. Since $\Gamma_{c}^{1}$ is closed in $\Gamma$ and the group $\Pi$ is contained in $\Pi^{*}$ by assumption, we have $\Pi^{*} \cong \mathbb{R}^{s}$ for some $s>0$. Moreover, the fix-double-loop $\mathscr{F}_{\Pi^{*}}$ is four-dimensional by Lemma 3.3 , since $\mathscr{F}_{\Pi^{*}} \neq \mathscr{F}_{\Xi}$. The group $\Pi^{*}$ is a normal subgroup of $\Gamma_{c}^{1}$, because $\Xi$ is normal in $\Gamma$. Thus the product $\Xi \Gamma_{c}^{1}$ leaves the Baer double loop $\mathscr{F}_{\Pi^{*}}$ invariant and by Lemma 3.2 and [3] we obtain the inequality

$$
\operatorname{dim} \Xi \Gamma_{c}^{1}=\operatorname{dim} \Xi \Gamma_{c}^{1} \mid \mathscr{F}_{\Pi^{*}}+\operatorname{dim} d^{\Xi \Gamma_{c}^{1}} \leqslant 4+7=11
$$

for some element $d \in c^{\Gamma} \backslash \mathscr{F}_{\Pi^{*}}$ Using the (topological) isomorphism $\Xi \Gamma_{c}^{1} / \Xi \cong \Gamma_{c}^{1} / \Xi \cap \Gamma_{c}^{1}$ this yields the inequality

$$
\operatorname{dim} \Gamma_{c}^{1}=\operatorname{dim} \Xi \Gamma_{c}^{1}-\operatorname{dim} \Xi+\operatorname{dim}\left(\Xi \cap \Gamma_{c}^{1}\right) \leqslant 11-t+s .
$$


Since $\operatorname{dim} \Gamma \geqslant 17$ and $\operatorname{dim} c^{\Gamma} \leqslant 7$, we have $\operatorname{dim} \Gamma_{c}^{1} \geqslant 10$, and therefore $t-s \leqslant 1$ holds. If the orbit $d^{\Xi}$ generates $\mathscr{D}$ for some element $d \in c^{\Gamma}$, then the stabilizer $\Xi_{d}$ is trivial and thus $\Xi_{c}=\Xi_{d^{\delta}}=\left(\Xi_{d}\right)^{\delta}=1$ for some automorphism $\delta \in \Gamma$ satisfying $d^{\delta}=c$. But this would contradict the fact that $1 \neq \Pi \leqslant \Xi_{c}$. Consequently, we have $\operatorname{dim}\left\langle d^{\Pi^{*}}\right\rangle=4$ for every $d \in c^{\Gamma} \backslash \mathscr{F}_{\Pi^{*}}$. Furthermore, the group $\Pi^{*}$ acts effectively on $\left\langle d^{\Pi^{*}}\right\rangle$, because $\mathscr{D}$ is generated by $\mathscr{F}_{\Pi^{*}}$ and the element $d$. Since $\Pi^{*}$ is isomorphic to $\mathbb{R}^{s}$, this implies that $s \leqslant 3$ by $[3,(2.4)]$. Thus we have $t \leqslant 1+s \leqslant 4$. Let $\mathrm{P}$ denote a closed one-parameter subgroup of $\Pi^{*}$. Since $\operatorname{dim} \mathscr{F}_{\Pi^{*}}=4$, we have $\mathscr{F}_{\mathrm{P}}=\mathscr{F}_{\Pi^{*}}$ by Lemma (3.3). Therefore, the centralizer $\Theta:=\mathrm{C}_{\Gamma} \mathrm{P}$ leaves $\mathscr{F}_{\mathrm{p}}$ invariant, and as before we infer that $\operatorname{dim} \Theta \leqslant 11$. Finally, considering the action of $\Gamma$ on the space of all one-dimensional subspaces of $\Xi$ we obtain that

$$
\operatorname{dim} \Gamma \leqslant \operatorname{dim} \Theta+\operatorname{dim} \Xi \leqslant 11+4 \leqslant 15
$$

which contradicts our assumption $\operatorname{dim} \Gamma \geqslant 17$.

Case 2. The element $c$ is moved by the vector group $\Pi$. Then the double loop $\mathscr{H}:=\left\langle c^{\Pi}\right\rangle$ is four-dimensional. Since $\Gamma_{c}^{1}$ acts on $\Pi$ and fixes the element $c$, it leaves $\mathscr{H}$ invariant. This implies that

$$
\operatorname{dim} \Gamma_{c}^{1}=\left.\operatorname{dim}\left(\Gamma_{c}^{1}\right)\right|_{\mathscr{H}}+\operatorname{dim} d^{\Gamma_{c}^{1}} \leqslant 4+7=11
$$

for some $d \in c^{\Gamma} \backslash \mathscr{H}$. Let $\mathrm{N} \leqslant \Gamma_{c}^{1}$ denote the kernel of the action of $\Gamma_{c}^{1}$ on $\Pi$ and set $\Lambda:=\Gamma_{[\mathscr{H}]}$. Then we have $\mathrm{N} \leqslant \Lambda$. In the following we shall show that in fact $\mathrm{N}=\Lambda$. For this, we first verify that $c^{\pi} \neq c$ holds for all $\pi \in \Pi \backslash\{1\}$. By Lemma 3.3, either $c^{\pi} \neq c$ or $\mathscr{F}_{\pi}=\mathscr{H}$ holds for any $\pi \in \Pi \backslash\{1\}$. Consider the set $\Pi^{*}:=\left\{\pi \in \Pi \mid \mathscr{H} \leqslant \mathscr{F}_{\pi}\right\}$. Using the same arguments as in the proof of Proposition 2.2 we infer that $\Pi^{*}=1$ and hence $c^{\pi} \neq c$ holds for all $\pi \in \Pi \backslash\{1\}$. Now we are able to prove the inclusion $\Lambda \subseteq N$. For this, choose an element $\lambda \in \Lambda$. Suppose that there is an element $\pi \in \Pi$ with $\lambda^{-1} \pi \lambda \neq \pi$. Then $1 \neq \vartheta:=\lambda^{-1} \pi \lambda \pi^{-1} \in \Pi$, and from the preceding arguments we infer that $c^{\vartheta} \neq c$. On the other hand, we have $c^{\vartheta}=c^{\lambda-1 \pi \lambda \pi^{-1}}=c^{\pi \lambda \pi^{-1}}$, and since $\lambda \in \Lambda$ and $c^{\pi} \in \mathscr{H}$, we conclude that $c \neq c^{\vartheta}=c^{\pi \lambda \pi^{-1}}=\left(c^{\pi}\right)^{\lambda \pi^{-1}}=\left(c^{\pi}\right)^{\pi^{-1}}=c$, which is a contradiction. This proves the equation $N=\Lambda$.

Now, the quotient group $\Gamma_{c}^{1} / \mathrm{N}=\Gamma_{c}^{1} / \Lambda$ acts effectively and irreducibly on $\Pi$, since $\Pi$ has been chosen to be minimal $\Gamma_{c}^{1}$-invariant. By $[8, \S 19.14, \S 19.17]$ this implies that there is a semi-simple linear Lie group $\Sigma$ (possibly $\Sigma=1$ ) such that $\Sigma \leqslant \Gamma_{c}^{1} / N \leqslant \Sigma \mathbb{C}^{\times}$. Thus either 
$\Gamma_{c}^{1} / N \leqslant \mathbb{R}$ or $\Gamma_{c}^{1} / N$ contains a torus subgroup. In the second case, the involution $\omega$ which is contained in the torus subgroup induces an involution $\omega^{*}:=\left.\omega\right|_{\mathscr{H}}$ on $\mathscr{H}$ (note that $\mathrm{N}=\Lambda$ ). By [3, (3.3)], we have $1 \leqslant \operatorname{dim} \mathscr{F}_{\omega^{*}} \leqslant 2$ and the desired inequality $\operatorname{dim} \Gamma \leqslant 16$ follows from Proposition (2.2). So it remains to study the case where $\Gamma_{c}^{1} / N \leqslant \mathbb{R}$. In this case, however, we immediately obtain that

$$
\operatorname{dim} \Gamma_{c} \leqslant \operatorname{dim} N+1=\operatorname{dim} \Lambda+1=\operatorname{dim} d^{\Lambda}+1 \leqslant 7+1=8
$$

for every element $d \in c^{\Gamma} \backslash \mathscr{H}$, and thus we have

$$
\operatorname{dim} \Gamma=\operatorname{dim} c^{\Gamma}+\operatorname{dim} \Gamma_{c} \leqslant 7+8=15,
$$

which finishes the proof.

\section{Appendix}

3.1. Lemma. Let $\mathscr{D}$ be a locally compact connected double loop of arbitrary finite (covering) dimension $n$. Let $U$ be a compact neighborhood in $\mathscr{D}$. Then the following statements hold:

(a) $\operatorname{dim} U=$ ind $U=$ Ind $U=\operatorname{dim}_{L} U=n$ for every principal ideal domain $L$, where $\operatorname{dim}_{L}$ denotes the cohomological dimension with coefficient domain $L$.

(b) The relation $\operatorname{dim}(U \times X)=\operatorname{dim} U+\operatorname{dim} X$ holds for every, locally compact paracompact space $X$.

Proof. The first assertion follows from Theorem 15.7 of [5] and from the fact that the covering dimension and the small and large inductive dimension coincide for a separable complete metric space, see [9, XI.1.2]. The second assertion follows from the equation $\operatorname{dim} U=\operatorname{dim}_{\mathbb{Z}_{p}} \mathscr{D}$ which holds for every prime number $p$ by part (a), and from a theorem of Y. KODAMA in $[13$, p. 400].

3.2. Lemma. Let $\mathscr{D}$ be a locally compact connected double loop of arbitrary finite dimension $n$. Let $\mathscr{H}$ be a closed sub-double-loop of $\mathscr{D}$ which is invariant under a locally compact automorphism group $\Gamma$ of $\mathscr{D}$. The kernel of the action of $\Gamma$ on $\mathscr{H}$ is denoted by $\Lambda$. Then the quotient group $\Delta=\Gamma / \Lambda$ of $\Gamma$ is a topological transformation group on $\mathscr{H}$ with respect to the quotient topology $\tau_{Q}$, and $\operatorname{dim}\left(\Delta, \tau_{Q}\right)=\operatorname{dim}\left(\Delta, \tau_{c o}\right)$, where $\tau_{c o}$ denotes the compact-open topology on $\Delta$ with respect to the action of $\Delta$ on $\mathscr{K}$. 
Proof. Let $\Phi: \mathscr{H} \times \Gamma \rightarrow \mathscr{H}$ be the evaluation mapping. Since $\mathscr{H}$ is locally compact, the compact-open topology on $\Delta$ is the coarsest topology such that the mapping $\Phi$ is continuous. Thus the topology $\tau_{Q}$ on $\Delta$ is finer than the topology $\tau_{c o}$, since the group $\left(\Delta, \tau_{Q}\right)$ evidently is a topological transformation group on $\mathscr{H}$. But his means that the identity mapping id: $\left(\Delta, \tau_{c o}\right) \rightarrow\left(\Delta, \tau_{Q}\right)$ is continuous. Since $\Gamma$ is locally compact by [2], we may select a compact neighborhood $U$ of the identity $\mathbb{1}$ in $\left(\Delta, \tau_{c o}\right)$. The restriction id $\left.\right|_{U}:\left(U, \tau_{c o}\right) \rightarrow\left(U, \tau_{Q}\right)$ is a homeomorphism. Hence we conclude that

$$
\begin{aligned}
\operatorname{dim}\left(\Delta, \tau_{c o}\right) & =\operatorname{ind}\left(\Delta, \tau_{c o}\right)=\operatorname{ind}\left(U, \tau_{c o}\right)=\operatorname{ind}\left(U, \tau_{Q}\right)= \\
& =\operatorname{ind}\left(\Delta, \tau_{Q}\right)=\operatorname{dim}\left(\Delta, \tau_{Q}\right),
\end{aligned}
$$

and the lemma is proved.

3.3. Lemma. If $1 \neq \Phi \unlhd \Gamma$ is a connected normal subgroup, then $\mathscr{F}_{\Phi}=\mathscr{F}_{\Gamma}$ or $\mathscr{F}_{\Phi}$ is four-dimensional. If $1 \neq \gamma \in \Gamma$ and $\operatorname{dim} C \geqslant 1$, where $C:=\left(C_{\Gamma} \gamma\right)^{\mathbf{1}}$, then $\mathscr{F}_{\gamma}=\mathscr{F}_{C}$ or $\mathscr{F}_{\gamma}$ is four-dimensional.

Proof. Since $\Phi$ is a normal subgroup of $\Gamma$, the fix-double-loop $\mathscr{F}_{\Phi}$ is $\Gamma$-invariant. If $\mathscr{F}_{\Phi} \neq \mathscr{F}_{\Gamma}$, then $\Gamma$ acts non-trivially on $\mathscr{F}_{\Phi}$. Because the group $\Gamma$ is connected, this implies that $\operatorname{dim} \mathscr{F}_{\Phi} \geqslant 4$ and thus $\mathscr{F}_{\Phi}$ is four-dimensional. Similarly, the connected group $C$ acts on $\mathscr{F}_{\gamma}$ and the claim follows as before.

\section{References}

[1] BöDi, R.: On the embedding of zero-dimensional double loops in locally euclidean double loops. Resultate der Math. 22, 657-666 (1992).

[2] BöDI, R.: Automorphism groups of locally compact connected double loops are locally compact. Arch. Math. 63, 291-294 (1993).

[3] BöbI, R.: On the dimensions of automorphism groups of 4-dimensional double loops. (to appear in Math. Z.).

[4] BOREL, A.: Seminar on transformation groups. Ann. of Math. Stud. 46, Princeton: Univ. Press. 1960.

[5] Bredon, G. E.: Sheaf Theory. New York: McGraw-Hill. 1967.

[6] Cartan, H., Eilenderg, S.: Homological Algebra. Princeton: Univ. Press. 1956.

[7] DoLD, A.: Lectures on Algebraic Topology. Berlin-Heidelberg-New York: Springer. 1972.

[8] Freudenthal, H., DE VRIEs, H.: Linear Lie Groups. New York-London: Academic Press. 1969.

[9] GrundhöFer, T., SalzmanN, H.: Locally compact double loops and ternary fields. In: Chein, O., Pflugfelder, H. D., Smith, J. D. H., (eds.) Quasigroups and Loops: Theory and Applications. pp. 313-356. Berlin: Heldermann. 1990.

[10] HALDeR, H. R.: Dimension der Bahn lokal kompakter Gruppen. Arch. Math. 22, 302-303 (1971).

[11] Hewitr, E., Ross, K. A.: Abstract Harmonic Analysis I, 2nd edn. Berlin-HeidelbergNew York: Springer. 1979. 
[12] IWASAWA, K.: On some types of topological groups. Ann. of Math. 50, 507-558 (1949).

[13] Kodama, Y:: A necessary and sufficient condition under which $\operatorname{dim} X \times Y=\operatorname{dim} X+$ $+\operatorname{dim}$ Y. Proc. Japan Acad. 36, 400 404 (1960).

[14] LöwEN, R.: Topology and dimension of stable planes: On a conjecture of $H$. Freudenthal. J. Reine Angew. Math. 343, 108-122 (1983).

[15] MASSEY, W. S.: Singular Homology Theory. Berlin-Heidelberg-New York: Springer. 1980.

[16] MonTGOMERY, D., ZIPPIN, L.: Topological Transformation Groups. New York: Wiley. 1955.

[17] Mostow, G. D.: The extensibility of local Lie groups of transformations. Ann. of Math. 52, 606-636 (1950).

[18] NaGAMI, K.: Dimension-theoretical structure of locally compact groups. J. Math. Soc. Japan 14, 379-396 (1962).

[19] Pears, A. R.: Dimension theory of general spaces. Cambridge: University Press. 19.75.

[20] Salzmann, H.: Topological planes. Adv. Math. 2, 1-60 (1967).

[21] SalzmanN, H.: Automorphismengruppen achtdimensionaler Ternärkörper. Math. Z. 166, 265-275 (1979).

[22] SalzmanN, H.: Compact 8-dimensional projective planes with large collineation groups. Geom. Dedicata 8, 139-161 (1979).

[23] Smith, P. A.: New results and old problems in finite transformation groups. Bull. Amer. Math. Soc. 66, 401-415 (1960).

[24] Spanier, E. H.: Algebraic Topology. New York: McGraw-Hill. 1966.

[25] VöLKLEIN, H.: Transitivitätsfragen bei linearen Liegruppen. Arch. Math. 36, 23-34 (1981).

RICHARD BöDI

Mathematisches Institut

Universität Tübingen

Auf der Morgenstelle 10

D-72076 Tübingen

Federal Republic of Germany

mmisa01@mailserv.zdv.uni-tuebingen.de 